\title{
Microchannels Effective Method for the Extraction of Oleuropein Compared with Conventional Methods
}

\author{
Mahnaz Yasemi, ${ }^{1}$ Amir Heydarinasab, ${ }^{1}$ Masoud Rahimi, ${ }^{2}$ and Mehdi Ardjmand ${ }^{3}$ \\ ${ }^{1}$ Department of Chemical Engineering, Science and Research Branch, Islamic Azad University, Tehran, Iran \\ ${ }^{2}$ CFD Research Center, Department of Chemical Engineering, Razi University, Taghe Bostan, 67149 Kermanshah, Iran \\ ${ }^{3}$ Department of Chemical Engineering, Islamic Azad University, South Tehran Branch, Tehran, Iran \\ Correspondence should be addressed to Amir Heydarinasab; a.heidarinasab@srbiau.ac.ir
}

Received 7 March 2017; Revised 7 May 2017; Accepted 14 May 2017; Published 8 June 2017

Academic Editor: Ana B. Martin-Diana

Copyright (C) 2017 Mahnaz Yasemi et al. This is an open access article distributed under the Creative Commons Attribution License, which permits unrestricted use, distribution, and reproduction in any medium, provided the original work is properly cited.

\begin{abstract}
Different methods of oleuropein extraction from olive leaf were investigated, including maceration, soxhlet, ultrasonic-assisted extraction, and microchannel. In current research, a response surface methodology (RSM) was used for prediction of the optimal values of parameters affecting the extraction of oleuropein through two methods of ultrasound and microchannel. Frequency $(F)$, temperature $(T)$, and power of ultrasound $(P)$ were the parameters which were studied in ultrasound method, but in microchannel system effects of $\mathrm{pH}$ and temperature $(T)$, volumetric flow rate ratio of two phases (VR), and contact time (CT) of two phases were optimized. UV detector device at $254 \mathrm{~nm}$ was used to recognize oleuropein through comparison of the retention time of the extracts with standard compound in chromatogram. The analysis of extracts was performed using HPLC. Optimum conditions for ultrasound were obtained as follows: $F=80 \mathrm{kHz}, T=25^{\circ} \mathrm{C}$, and $P=100 \mathrm{w}$. Using these optimum conditions, the extraction of oleuropein was $81.29 \%$. Amount of oleuropein extraction by microchannel method in optimum conditions was $96.29 \%$, which was way more than other applied methods. Microchannel system as a continuous method has many advantages including low solvent consumption, being environment friendly, short time for extraction, and high efficiency.
\end{abstract}

\section{Introduction}

Olive leave as a photochemical source of phenol and flavonoid [1] has antioxidant [2-6] and antibacterial [7-10] properties. Highest amount of phenol derivatives in olive leaf is oleuropein, whose amount depends on harvest time and varies between 17 and 23\% [11]. Main compound of biophenol in olive is oleuropein which is widely studied, because it has wide spectrum of health applications in diseases such as blood pressure [12], heart disease [13], bacterial infections, viral infections [14], and cancer [15, 16]. Amount of oleuropein in ripe fruit is more than growing fruit [17]. Different factors such as extraction method, type of solvent, temperature, and extraction time affect efficiency. Maceration, soxhlet, supercritical fluids [18], ultrasound [19], and liquid-liquid extraction [20] can be used for extraction procedure. Extraction efficiency of traditional methods is low and much solvent is needed; therefore damage can be caused to environment [21].
There is a growing pressure on different industries to use modern methods that are safe for human being and environment. Green chemistry is one of important and known subjects for encouraging design and production of harmless and environment friendly products. Using microchannels for extracting compounds of medicinal herbs is an appropriate replacement for traditional methods and is a new hope for achieving green chemistry [22].

The present study is aimed at extracting the oleuropein from olive leaves via diverse extraction approaches and to compare these approaches together. Another goal of this study is to develop and optimize a green, easy, reliable, repeatable, and inexpensive process for extraction of oleuropein as an important phenolic compound from olive leaf. In current study, three main factors of $F, T$, and $P$ for ultrasonic bath, and four parameters of $\mathrm{VR}, \mathrm{pH}, T$, and $\mathrm{CT}$ for microchannel were chosen to be optimized with RSM based on a central composite design. Liquid-liquid extraction using microchannel method is an economic and continuous method with low 
energy and solvent consumption that requires short time, which can play an important role in various food processing, pharmaceutical, and cosmetics industries. The coil inside the microchannel is used for the first time to extract oleuropein from olive leaves. Because the coil creates turbulence and prevents the formation of the boundary layer, therefore it increases the mass transfer, separation, and efficiency.

\section{Materials and Methods}

2.1. Samples Preparation. Olive leaves were collected from Ilam province in October 2015. Samples were washed with deionized water and leaves were dried immediately in a shady and well-aired place for two weeks. Dried leaves were powdered using an electric mill (IKA, Germany). Then leaves were powdered and stored in dark and cold place until use.

2.2. Chemicals. All chemicals used in current study such as oleuropein (purity $\geq 98 \%$ by HPLC), chromatographic grade-double distilled water, and HPLC grade acetonitrile, ethyl acetate, ethanol absolute, methanol, sodium hydroxide, and phosphoric acid were purchased from Merck Company (Darmstadt, Germany).

\section{Experimental}

3.1. HPLC Analysis. In order to determine oleuropein of olive leaf extract, reversed phase high-performance liquid chromatography (HPLC) technique was applied with silicabased $\mathrm{C} 18$ bonded phase column $(250 \mathrm{~mm} \times 4.6 \mathrm{~mm} \mathrm{ID}$, $3 \mu \mathrm{m}$ particle size) with mobile phase containing a mixture of phosphate buffer and acetonitrile ( $80: 20$ volume ratio) at a flow rate of $1 \mathrm{~mL} / \mathrm{min}$. Oleuropein was determined at $254 \mathrm{~nm}$ using a UV detector. The injection volume of both solutions of standard and sample is $1 \mu \mathrm{L}$. The HPLC system (KNAUER, Germany) consisting of dual pumps, vacuum degasser, and system controller was used.

3.2. Maceration Method. An amount of $10 \mathrm{~g}$ of olive leaves powder was analytes soaked in $100 \mathrm{~mL}$ of diverse solvents. The obtained mixture was magnetically stirred for $72 \mathrm{~h}$. Then, filtration of extracts was carried out using a Whatman No. 1 filter and it was analyzed using HPLC.

3.3. Soxhlet Extraction. An amount of $30 \mathrm{~g}$ of olive leaves sample was put in the thimble of soxhlet device and extracted using $300 \mathrm{~mL}$ of divers' solvents during 8 hours. Extracts were cooled until ambient temperature, filtered, and measured using HPLC.

3.4. Ultrasonic-Assisted Extraction. A compound containing $10 \mathrm{~g}$ of sample and solvent was transferred to $100 \mathrm{~mL}$ beaker and placed in ultrasound bath such that liquid level was lower than ultrasound bath medium. Effects of temperature, frequency, and power of ultrasound were investigated on extraction efficiency. Obtained essence was rinsed by filter paper and then analyzed by HPLC.
3.5. Microchannel Extraction. T-shaped microchannel mixer is the key part of this device. The inner diameter and length Tshaped microchannel were $600 \mu \mathrm{m}$ and $70 \mathrm{~mm}$, respectively. A coil with an outer diameter of $2 \mathrm{~mm}$, an inner diameter of $600 \mu \mathrm{m}$, and length of $700 \mathrm{~mm}$ was applied in the outlet stream for enhancing the time of residence. The syringe pumps were applied for diverting feed in all inlet streams. The microchannel was put in a water bath for keeping its temperature fixed at the chosen range over the trial procedure. HPLC system was used to determine the oleuropein level after separation of two phases in the outlet stream. The influence of several factors including $\mathrm{pH}$, temperature, and volumetric flow rate ratio and contact time was studied to achieve the best extraction conditions. The obtained outcomes were presented in extraction recovery percentages and compared with other procedures (Figure 1).

With respect to the fact that selecting proper solvent has significant effect on efficiency and extraction quality, in microchannel method, $10 \mathrm{~g}$ of olive leave powder was mixed with $100 \mathrm{~mL}$ of different organic phase including butanol, hexane, and ethyl acetate and, after filtration, one syringe pump was filled with the mixture. Second syringe should be filled with aqueous phase. For selecting proper aqueous phase each time syringe was filled with different solvents including ethanol absolute ( $80 \%$ and $20 \%$ ), methanol (80\% and $20 \%)$, and ethyl acetate and connected into microfluidic system. At the end of extraction, aqueous phase was injected into HPLC. Based on experiment, results of ethyl acetate were selected as organic phase.

\section{Results and Discussion}

4.1. Optimization of Solvent Type. In order to study the impact of extraction solvent on content of oleuropein, constant amounts of olive leaves were extracted using divers solvents. The extraction of samples was performed using $100 \%$ methanol, $20 \%$ methanol, water, $20 \%$ ethanol absolute, $100 \%$ ethanol absolute, and ethyl acetate, respectively. For selecting the best solvent in each method, the following procedure should be followed: in maceration method, at each run, $10 \mathrm{~g}$ of olive leave described in preparation stage should be separately mixed with different solvents including ethanol absolute ( $80 \%$ and $20 \%$ ), methanol ( $80 \%$ and $20 \%)$, and ethyl acetate. After 72 hours, extract should be filtered and injected in HPLC for measuring oleuropein. The results of extraction were used to help choose the best solution. As described in soxhlet extraction method, ethanol absolute, methanol, and ethyl acetate were used separately, and extracts were filtered. Extract was injected into HPLC for measuring oleuropein content. In ultrasonic method, $10 \mathrm{~g}$ of sample was mixed separately with aforementioned solvents and placed in ultrasonic bath with frequency of $80 \mathrm{kHz}$ and power of 70 watts for 10 minutes. Extract was filtered and injected in HPLC for measuring extract content.

In microchannel method, $10 \mathrm{~g}$ of olive leave powder was mixed with $100 \mathrm{~mL}$ of ethyl acetate and after filtration one syringe pump was filled with the mixture. Second syringe should be filled with aqueous phase. For selecting proper aqueous phase each time syringe was filled with 


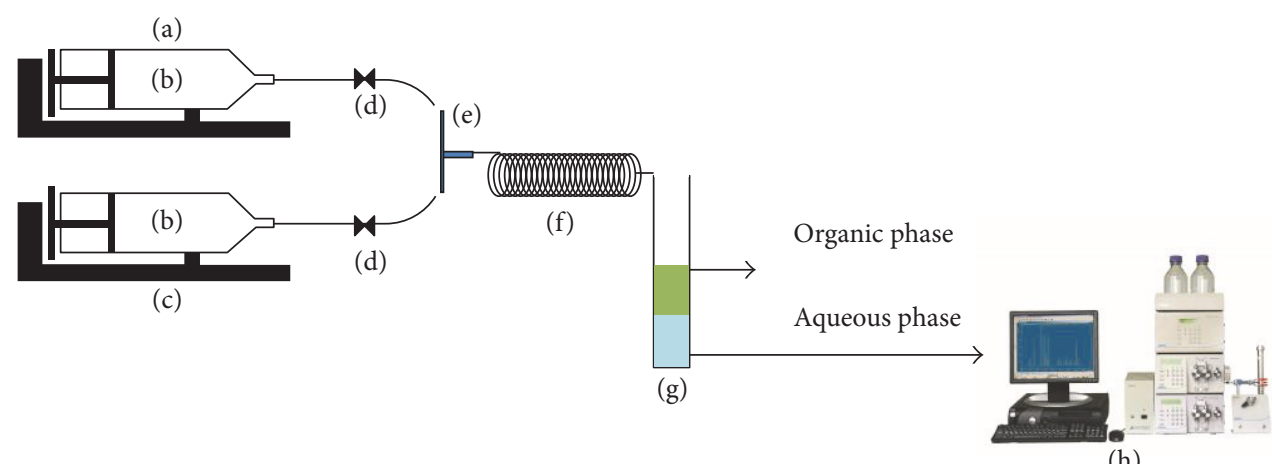

Figure 1: Microchannel system: (a) organic phase, (b) microsyringe pumps, (c) aqueous phase, (d) valve, (e) T-shaped microchannel, (f) coil, (g) extracted sample, and (h) HPLC.

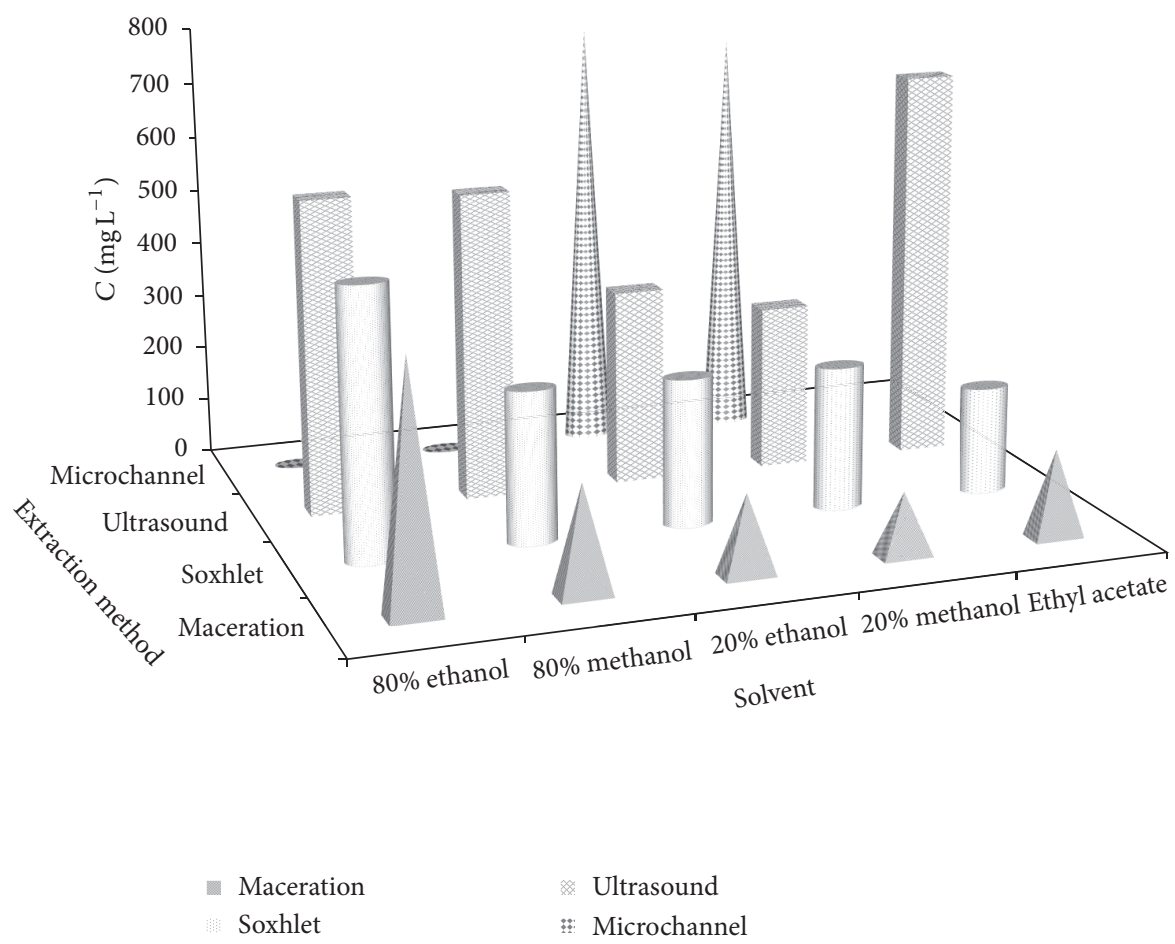

FIGURE 2: Effects of different solvents on the extraction oleuropein for maceration, soxhlet, ultrasound, and microchannel.

different solvents including ethanol absolute ( $80 \%$ and $20 \%)$, methanol ( $80 \%$ and $20 \%)$, and ethyl acetate and connected into microfluidic system. At the end of extraction, aqueous phase was injected into HPLC. Results of selecting optimum solvent are presented in Figure 2.

According to Figure 2, the maximum extract content of $779.49 \mathrm{mg} / \mathrm{L}$ and $707.41 \mathrm{mg} / \mathrm{L}$ was achieved in microchannel and UAE methods, respectively.

4.2. Statistical Analysis. Data was analyzed using DesignExpert (Stat-Ease, trial version) [23]. The number of required experiments was reduced by using RSM and so the cost of experiment was also reduced. Then, this methodology was used to investigate the influence of multiple factors and their interaction. RSM, as a powerful statistical method to optimize complex procedures, has been applied for optimizing the extraction of numerous molecules like protein [24], polysaccharides [25], and antioxidant [26], and phenols compounds [27]. In current research, RSM was used for optimization of extracting oleuropein from olive leaves using ultrasoundassisted extraction and microchannel system.

4.3. Model Fitting for Ultrasound-Assisted Extraction. The optimization of oleuropein extraction via ultrasonic bath was performed with the RSM. For this purpose, a central composite design (CCD) was used. Ultrasonic frequency, ultrasonic power, and temperature were considered as independent extraction variables. The range and center point values relating to these three independent variables have been given in Table 1 considering the obtained outcomes of 
TABLE 1: Independent variables and their coded levels used in RSM studies for USA method.

\begin{tabular}{lccc}
\hline Factors & Units & Low level $(-1)$ & High level $(+1)$ \\
\hline Frequency $(F)$ & $\mathrm{kHz}$ & 20 & 80 \\
Power $(P)$ & $\mathrm{W}$ & 40 & 100 \\
Temperature $(T)$ & ${ }^{\circ} \mathrm{C}$ & 25 & 75 \\
\hline
\end{tabular}

preliminary tests. Numbers of 20 runs were performed with RSM design which the related design, experiential responses, and the predicted values for are shown in Table 2.

The obtained outcomes indicate excellent fitting of the experimental and theoretical models. Considering the multiple regression analysis performed on the experimental data, the following second-order polynomial equation can be used to describe the relationship between the response and the test variables.

$$
\begin{aligned}
y= & 66.77+4.63 * A+2.21 * B+10.92 * C+2.21 \\
& * A * B-2.03 * A * C-6.39 * B * C-5.07 \\
& * A^{2}+6.06 * B^{2}-1.76 * C^{2}
\end{aligned}
$$

where $A=F(\mathrm{kHz}), B=T\left({ }^{\circ} \mathrm{C}\right)$, and $C=P(\mathrm{w})$.

The analysis of variance (ANOVA) outcomes are given in Table 3. Based on ANOVA of the quadratic regression model, $R^{2}$ coefficient was 0.9661 and the adjusted determination coefficient (Adj. $R^{2}$ ) was 0.9356 that indicates the ability of model to describe $96.61 \%$ variability for the response variable. Moreover, the lack of fit usually applied for measuring the model was not significant as its $P$ value was 0.3658 $(>0.05)$, which showed excellent fit of the model with this experimental project. Values of Prob. $>F$ of $<0.05$ showed that the terms of model were significant. In this situation, $A, B, C, A B, B C, A^{2}$, and $B^{2}$ were significant model terms. The maximum response value for extraction recovery $81.29 \%$ was estimated using software of Design-Expert as frequency of $80 \mathrm{kHz}$, power of $100 \mathrm{w}$, and temperature of $25^{\circ} \mathrm{C}$. Actual efficiency of extraction under these conditions was $80.67 \pm$ $0.62 \%$ which is close to the predicted value, which indicated the suitability of model for extraction process.

4.4. Model Fitting for Microfluidic Extraction Process. There are numerous parameters affecting quantity and quality of the tannin extracts. The experiments were designed using the software of Design-Expert. RSM has been known as a useful method to optimize experimental conditions for maximizing different responses. The CCD is a RSM design used for finding the optimal experimental condition with certain values for main experimental measurements for the highest recovery of oleuropein in olive leaves extract. These variables included volumetric flow rate ratio of flow, contact time, $\mathrm{pH}$, and temperature. Three levels of $-1,0$, and +1 are available for each factor. A total number of 30 runs of experiments were carried out for determining important parameters in extraction of oleuropein, as can be observed in Table 4 .
The equation of predictive model of extraction recovery is described as follows:

$$
\begin{aligned}
y= & 60.37-9.37 A+0.86 B-4.33 C-9.667 \times 10^{-3} D \\
& -3.03 A * B-3.65 A * C+7.25 A * D-3.69 B \\
& * C-6.89 B * D-8.76 C * D+6.77 A^{2} \\
& +0.077 B^{2}+5.90 C^{2}+2.17 D^{2},
\end{aligned}
$$

where $A=F R, B=R T, C=\mathrm{pH}$, and $D=T$.

The response is greater and smaller at the high level when a parameter has a positive and negative influence, respectively. The important second-order polynomial model equation at level of $5 \%$ to optimize the extraction efficiency of oleuropein with microfluidic system is similar to equation (2). Table 5 demonstrates the result of ANOVA of quadratic regression model for oleuropein recovery. The high $F$-value and nonconsiderable lack of fit show that the acquired experimental data were excellently fitted with the model. Linear parameters including volumetric flow rate ratio $(C)$ and temperature $(A)$ had negative coefficients, while contact time $(B)$ and $\mathrm{pH}(D)$ had positive coefficients. Square factors including $A^{2}, B^{2}, C^{2}$, and $D^{2}$ exhibited positive coefficients. Negative coefficients were observed for quadratic or interaction parameters including $A B, A C, B C, B D$, and $C D$, while positive coefficient was observed for $A D$.

$P$ value for $A$ and $C$ and square effect factors called $A^{2}$ and $C^{2}$ as well as quadratic or interaction factors $A B, A C$, $A D, B C, B D$, and $C D$ were $<0.05$. $P$ values were applied for assessing the importance and influence of each parameter and the statistical polynomial model equation described that the greater the $F$-value magnitude and the lower the $P$ value, the more considerable the corresponding coefficient. Accordingly, finding of the current research indicated that square factors $B^{2}$ and $D^{2}$ terms were not important $(P>$ 0.05). Based on Table $5, R^{2}$ value was $97.85 \%$, which shows excellent fitness of the model. The higher the $R^{2}$ value toward unity is, the better the model fits the experimental data.

According to the outcomes for lack of fit, its $F$ and $P$ values were 2.21 and 0.1735 , respectively. The nonconsiderable $P$ value shows that the model was excellent and fitted with the experimental data very well. In order to confirm the model prediction, the best reaction conditions were used for three independent replicates for synthesizing biodiesel, and the mean \pm standard deviation was also reported. Figure 3 shows the correlation between the empirical and predicted data computed for extraction recovery of oleuropein using microfluidic system. It can be observed that the estimated date computed from the model is in excellent accordance with the empirical data in the working conditions range. The best condition for extracting oleuropein was $T=35^{\circ} \mathrm{C}$, VR $=$ $1.75, \mathrm{CT}=30 \mathrm{~s}$, and $\mathrm{pH}=2.23$. Using these conditions, the extraction recovery was obtained as $96.29 \pm 0.45 \%$ and the actual value was $95.47 \pm 0.25 \%$.

4.5. Comparison between Extraction Methods. Main stage in providing herbal products is selecting appropriate extraction 
TABLE 2: Experimental design recommended by Design-Expert for ultrasound-assisted extraction.

\begin{tabular}{|c|c|c|c|c|c|}
\hline Std order & Run order & $A=$ frequency & $B=$ temperature & $C=$ power of ultrasound & Yield\% \\
\hline 19 & 1 & 50 & 50 & 70 & 68.86 \\
\hline 18 & 2 & 50 & 50 & 70 & 63.31 \\
\hline 4 & 3 & 80 & 75 & 40 & 72.92 \\
\hline 16 & 4 & 50 & 50 & 70 & 63.43 \\
\hline 6 & 5 & 80 & 25 & 100 & 80.63 \\
\hline 1 & 6 & 20 & 25 & 40 & 40.25 \\
\hline 11 & 7 & 50 & 25 & 70 & 73.23 \\
\hline 17 & 8 & 50 & 50 & 70 & 66.71 \\
\hline 10 & 9 & 80 & 50 & 70 & 64.65 \\
\hline 3 & 10 & 20 & 75 & 40 & 55.14 \\
\hline 15 & 11 & 50 & 50 & 70 & 65.51 \\
\hline 13 & 12 & 50 & 50 & 40 & 54.23 \\
\hline 9 & 13 & 20 & 50 & 70 & 60.85 \\
\hline 8 & 14 & 80 & 75 & 100 & 78.79 \\
\hline 12 & 15 & 50 & 75 & 70 & 74.53 \\
\hline 14 & 16 & 50 & 50 & 100 & 77.89 \\
\hline 20 & 17 & 50 & 50 & 70 & 68.56 \\
\hline 5 & 18 & 20 & 25 & 100 & 79.82 \\
\hline 2 & 19 & 80 & 25 & 40 & 51.86 \\
\hline 7 & 20 & 20 & 75 & 100 & 66.49 \\
\hline
\end{tabular}

TABLE 3: ANOVA for response surface reduced quadratic model for USA method.

\begin{tabular}{|c|c|c|c|c|c|}
\hline Source & Sum of squares & $\mathrm{df}$ & Mean square & $F$ values & $P$ values Prob $>F$ \\
\hline Model & 1987.59 & 9 & 220.84 & 31.66 & $<0.0001$ \\
\hline$A$ & 214.37 & 1 & 214.37 & 30.73 & 0.0002 \\
\hline$B$ & 48.75 & 1 & 48.75 & 6.99 & 0.0246 \\
\hline C & 1192.90 & 1 & 1192.90 & 171.01 & $<0.0001$ \\
\hline$A B$ & 38.98 & 1 & 38.98 & 5.59 & 0.0397 \\
\hline$A C$ & 33.13 & 1 & 33.13 & 4.75 & 0.0543 \\
\hline$B C$ & 326.66 & 1 & 326.66 & 46.83 & $<0.0001$ \\
\hline$A^{2}$ & 70.64 & 1 & 70.64 & 10.13 & 0.0098 \\
\hline$B^{2}$ & 101.05 & 1 & 101.05 & 14.49 & 0.0035 \\
\hline$C^{2}$ & 8.50 & 1 & 8.50 & 1.22 & 0.2955 \\
\hline Residual & 69.76 & 10 & 6.98 & & \\
\hline Lack of fit & 40.46 & 5 & 8.09 & 1.38 & 0.3658 \\
\hline Pure error & 29.29 & 5 & 5.86 & & \\
\hline Cor total & 2057.35 & 19 & & & \\
\hline$R^{2}=0.9661$ & $R^{2}($ Pred $)=0.8199$ & & $R^{2}($ adj $)=0.9356$ & & \\
\hline
\end{tabular}

method, which affects quality and quantity of effective substance. Modern extraction methods result in higher amount of higher quality products. Current study is to improve extraction method using microchannel. Advantages of this method include high-speed, cost-effectiveness (in terms of time, energy, and equipment size), no need for auxiliary separation procedures, and high efficiency. Among modern extraction techniques, microfluidic system is more interesting than the ultrasound-assisted extraction as it enhances the mass transfusion via solid matrix of the plant substance, has quicker combination of the liquid, maintaining the maximum probable driving forces, and ensures the maximum quantity, quality, and purity of the active elements in the extract, which support our research. Comparison of the recoveries of extraction procedures is presented in Figure 4 given in percentage. According to these results, the soxhlet extraction technique with recovery of $62 \%$ had the maximum recovery between the conventional approaches and its corresponding modern technique was microchannel with yield of $95.47 \%$. Therefore, Design-Expert was employed in this study for 
TABLE 4: Experimental design recommended by Design-Expert for MC method.

\begin{tabular}{|c|c|c|c|c|c|c|}
\hline std & Run & $A=$ temperature $\left({ }^{\circ} \mathrm{C}\right)$ & $B=$ contact time $(\mathrm{s})$ & $C=$ volumetric flow rate ratio & $D=\mathrm{pH}$ & Yield\% \\
\hline 21 & 1 & 50 & 20.00 & 1.75 & 6.00 & 63.72 \\
\hline 9 & 2 & 50 & 20.00 & 1.75 & 6.00 & 65 \\
\hline 12 & 3 & 50 & 20.00 & 1.75 & 6.00 & 61.99 \\
\hline 14 & 4 & 65 & 15.00 & 2.38 & 8.00 & 61.23 \\
\hline 13 & 5 & 65 & 15.00 & 1.13 & 4.00 & 54 \\
\hline 17 & 6 & 35 & 25.00 & 2.38 & 8.00 & 73.52 \\
\hline 2 & 7 & 50 & 20.00 & 3.00 & 6.00 & 66.73 \\
\hline 7 & 8 & 35 & 15.00 & 1.13 & 4.00 & 72.1 \\
\hline 4 & 9 & 50 & 20.00 & 1.75 & 10.00 & 65.27 \\
\hline 16 & 10 & 50 & 10.00 & 1.75 & 6.00 & 60.21 \\
\hline 8 & 11 & 50 & 20.00 & 1.75 & 6.00 & 63 \\
\hline 20 & 12 & 35 & 15.00 & 2.38 & 4.00 & 80 \\
\hline 11 & 13 & 20 & 20.00 & 1.75 & 6.00 & 89 \\
\hline 22 & 14 & 50 & 20.00 & 0.50 & 6.00 & 71 \\
\hline 28 & 15 & 65 & 15.00 & 1.13 & 8.00 & 67.5 \\
\hline 23 & 16 & 35 & 25.00 & 1.13 & 4.00 & 85.2 \\
\hline 5 & 17 & 50 & 20.00 & 1.75 & 6.00 & 66 \\
\hline 26 & 18 & 65 & 25.00 & 1.13 & 8.00 & 65.47 \\
\hline 1 & 19 & 65 & 25.00 & 2.38 & 8.00 & 53.6 \\
\hline 25 & 20 & 50 & 20.00 & 1.75 & 2.00 & 65 \\
\hline 24 & 21 & 65 & 15.00 & 2.38 & 4.00 & 56.9 \\
\hline 15 & 22 & 50 & 20.00 & 1.75 & 6.00 & 63.1 \\
\hline 6 & 23 & 50 & 30.00 & 1.75 & 6.00 & 66 \\
\hline 30 & 24 & 50 & 20.00 & 1.75 & 6.00 & 62.6 \\
\hline 19 & 25 & 65 & 25.00 & 2.38 & 4.00 & 58.5 \\
\hline 10 & 26 & 35 & 15.00 & 1.13 & 8.00 & 76.7 \\
\hline 27 & 27 & 35 & 25.00 & 2.38 & 4.00 & 86.4 \\
\hline 29 & 28 & 65 & 25.00 & 1.13 & 4.00 & 60 \\
\hline 18 & 29 & 80 & 20.00 & 1.75 & 6.00 & 61 \\
\hline 3 & 30 & 35 & 15.00 & 2.38 & 8.00 & 75 \\
\hline
\end{tabular}

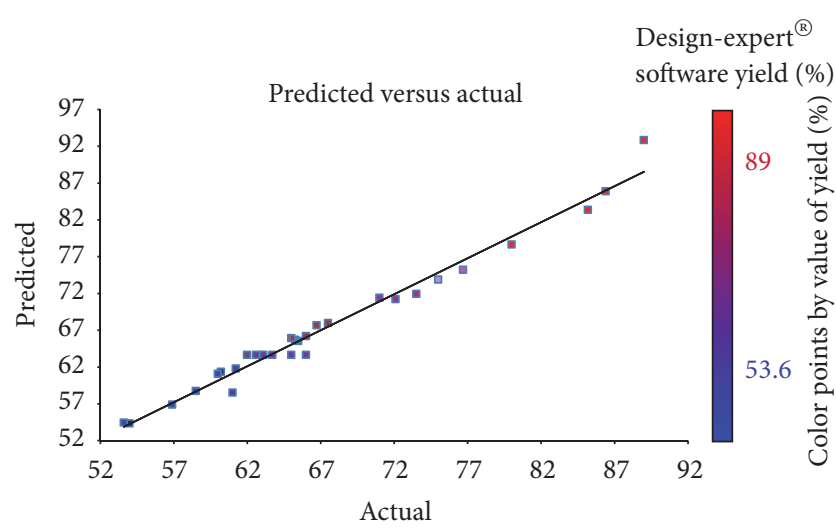

FIGURE 3: The correlation between the experimentally obtained values of extraction yield of oleuropein versus the calculated values using the model equation.

studying the influence of frequency, temperature, and power ultrasonic on the yield of extraction and for determining

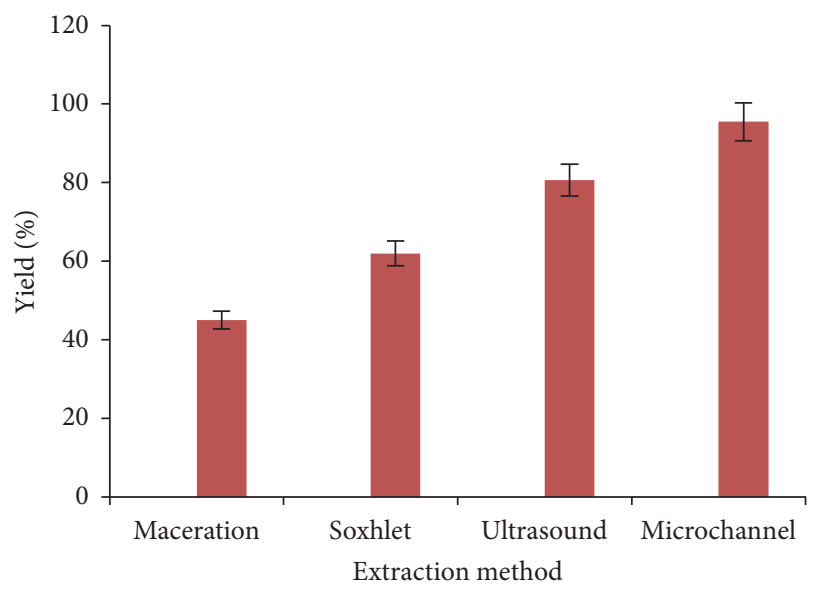

FIGURE 4: Comparison of methods of oleuropein extraction from olive leaf.

the optimal factors for maximizing recovery in microfluidic extraction process. At the best conditions, extraction yield 
TABLE 5: ANOVA for response surface reduced quadratic model for MC method.

\begin{tabular}{|c|c|c|c|c|c|}
\hline Source & Sum of squares & $\mathrm{df}$ & Mean square & $F$ values & $P$ values \\
\hline Model & 2339.95 & 14 & 167.14 & 48.72 & $<0.0001$ significant \\
\hline A-flow rate & 383.88 & 1 & 383.88 & 111.89 & $<0.0001$ significant \\
\hline$B$-residence time & 3.48 & 1 & 3.48 & 1.02 & 0.3297 not significant \\
\hline $\mathrm{C}-\mathrm{PH}$ & 60.70 & 1 & 60.70 & 17.69 & 0.0008 significant \\
\hline D-temperature & $3.031 \times 10^{-4}$ & 1 & $3.031 \times 10^{-4}$ & $8.833 \times 10^{-5}$ & 0.9926 not significant \\
\hline$A B$ & 25.31 & 1 & 25.31 & 7.38 & 0.0159 significant \\
\hline$A C$ & 20.58 & 1 & 20.58 & 6.00 & 0.0271 significant \\
\hline$A D$ & 81.31 & 1 & 81.31 & 23.70 & 0.0002 significant \\
\hline$B C$ & 21.05 & 1 & 21.05 & 6.14 & 0.0256 significant \\
\hline$B D$ & 73.36 & 1 & 73.36 & 21.38 & 0.0003 significant \\
\hline$C D$ & 66.66 & 1 & 66.66 & 19.43 & 0.0005 significant \\
\hline$A^{2}$ & 256.85 & 1 & 256.85 & 74.86 & $<0.0001$ significant \\
\hline$B^{2}$ & 0.034 & 1 & 0.034 & $9.782 \times 10^{-3}$ & 0.9225 not significant \\
\hline$C^{2}$ & 61.70 & 1 & 61.70 & 17.98 & 0.0007 significant \\
\hline$D^{2}$ & 8.33 & 1 & 8.33 & 2.43 & 0.1399 not significant \\
\hline Residual & 51.46 & 15 & 3.43 & & \\
\hline Lack of fit & 39.53 & 9 & 4.39 & 2.21 & 0.1735 not significant \\
\hline Pure error & 11.93 & 6 & 1.99 & & \\
\hline Cor total & 2391.41 & 29 & & & \\
\hline$R^{2}=97.85 \%$ & $R^{2}($ Pred $)=88.49 \%$ & $R^{2}(\mathrm{adj})=95.84 \%$ & Std. Dev $=1.85$ & & \\
\hline
\end{tabular}

was 95.47 and $80.67 \%$ for microchannel and ultrasonic bath, respectively.

\section{Conclusions}

According to results, microfluidic system for extracting oleuropein is better than other traditional methods such as maceration, soxhlet, and ultrasound. This method prevents thermal degradation and results in increase in bioavailability of bioactive substances. However, ultrasound method provides good performance but is expensive and using organic solvents makes the method harmful for environment.

Microchannel system is the new and the most hopeful method, which can be a better choice for extracting oleuropein from olive leaves at industrial scale as it has shorter procedure time, lower temperature, and high extract yield. This technique prevents the thermal degradation, enhances the bioactive components bioavailability, which increases antioxidant activity, and overcomes the drawback of other techniques. The developed technique like microfluidic system is fast, not complex, and easy to operate. In this study, the best conditions of extraction procedure were obtained using RSM at ultrasound frequency of $80 \mathrm{kHz}$, solvent power of $100 \mathrm{w}$, and temperature of $25^{\circ} \mathrm{C}$. Oleuropein obtained under the optimal conditions is more than that reported using conventional extraction approaches, such as soxhlet and maceration. The highest selectivity for the extraction can be obtained for extraction of oleuropein through the microfluidic extraction.

\section{Conflicts of Interest}

The authors declare that they have no conflicts of interest.

\section{References}

[1] S. Bulotta, M. Celano, S. M. Lepore, T. Montalcini, A. Pujia, and D. Russo, "Beneficial effects of the olive oil phenolic components oleuropein and hydroxytyrosol: focus on protection against cardiovascular and metabolic diseases," Journal of Translational Medicine, vol. 12, pp. 219-223, 2014.

[2] F. Visioli, S. Bellosta, and C. Galli, "Oleuropein, the bitter principle of olives, enhances nitric oxide production by mouse macrophages," Life Sciences, vol. 62, no. 6, pp. 541-546, 1998.

[3] F. Visioli and C. Galli, "Limited conformational flexibility of a disulfide-linked apolipoprotein A-I dimer in reconstituted high density lipoproteins," Atherosclerosis, vol. 135, p. S6, 1997.

[4] N. K. Andrikopoulos, S. Antonopoulou, and A. C. Kaliora, "Oleuropein inhibits LDL oxidation induced by cooking oil frying by-products and platelet aggregation induced by plateletactivating factor," LWT - Food Science and Technology, vol. 35, no. 6 , pp. 479-484, 2002.

[5] H. Jemai, M. Bouaziz, I. Fki, A. El Feki, and S. Sayadi, "Hypolipidimic and antioxidant activities of oleuropein and its hydrolysis derivative-rich extracts from Chemlali olive leaves," Chemico-Biological Interactions, vol. 176, no. 2-3, pp. 88-98, 2008.

[6] N. K. Andrikopoulos, F. N. Salta, A. Mylona, A. Chiou, and G. Boskou, "Oxidative stability of edible vegetable oils enriched in polyphenols with olive leaf extract," Food Science and Technology International, vol. 13, no. 6, pp. 413-421, 2007. 
[7] G. Bisignano, A. Tomaino, R. Lo Cascio, G. Crisafi, N. Uccella, and A. Saija, "On the in vitro antimicrobial activity of oleuropein and hydroxytyrosol," Journal of Pharmacy and Pharmacology, vol. 51, no. 8, pp. 971-974, 1999.

[8] P. M. Furneri, A. Marino, A. Saija, N. Uccella, and G. Bisignano, "In vitro antimycoplasmal activity of oleuropein," International Journal of Antimicrobial Agents, vol. 20, no. 4, pp. 293-296, 2002.

[9] D. Markín, L. Duek, and I. Berdícevsky, "In vitro antimicrobial activity of olive leaves," Mycoses, vol. 46, no. 3-4, pp. 132-136, 2003.

[10] A. P. Pereira, I. C. F. R. Ferreira, F. Marcelino et al., "Phenolic compounds and antimicrobial activity of olive (Olea europaea L. Cv. Cobrançosa) leaves," Molecules, vol. 12, no. 5, pp. 11531162, 2007.

[11] H. Yateem and I. Afaneh, "Optimum Conditions for Oleuropein Extraction from Olive Leaves," International Journal of Applied Science and Technology, vol. 4, p. 153, 2014.

[12] R. H. X. Wong, M. L. Garg, L. G. Wood, and P. R. C. Howe, "Antihypertensive potential of combined extracts of olive leaf, green coffee bean and beetroot: A randomized, double-blind, placebo-controlled crossover trial," Nutrients, vol. 6, no. 11, pp. 4881-4894, 2014.

[13] W. A. Pryor, "The antioxidant nutrients and disease preventionwhat do we know and what do we need to find out?" The American Journal of Clinical Nutrition, vol. 53, pp. 391S-393S, 1991.

[14] D. Fügen, T. Mücahit, and D. Özakaya, "Influence of the phenophase on the phenolic profile and antioxidant properties of dalmatian sage," Journal of Food Processing and Technology, vol. 2, pp. 3-8, 2011.

[15] R. Fabiani, A. De Bartolomeo, P. Rosignoli, M. Servili, G. F. Montedoro, and G. Morozzi, "Cancer chemoprevention by hydroxytyrosol isolated from virgin olive oil through G1 cell cycle arrest and apoptosis," European Journal of Cancer Prevention, vol. 11, no. 4, pp. 351-358, 2002.

[16] A. M. Kountouri, A. C. Kaliora, L. Koumbi, and N. K. Andrikopoulos, "In-vitro gastric cancer prevention by a polyphenol-rich extract from olives through induction of apoptosis," European Journal of Cancer Prevention, vol. 18, no. 1, pp. 33-39, 2009.

[17] I. Afaneh, H. Yateem, and F. Al-Rimawi, "Effect of Olive Leaves Drying on the Content of Oleuropein," American Journal of Analytical Chemistry, vol. 06, no. 03, pp. 246-252, 2015.

[18] S. Selin and M. Bilgin, "Aflatoxin species: their health effects and determination methods in different food stuffs," Sep. Sci. Technol, vol. 10, no. 3, pp. 675-685, 2012.

[19] R. Japón-Luján, J. M. Luque-Rodríguez, and M. D. Luque De Castro, "Dynamic ultrasound-assisted extraction of oleuropein and related biophenols from olive leaves," Journal of Chromatography $A$, vol. 1108, no. 1, pp. 76-82, 2006.

[20] I. G. Mekinić, M. Gotovac, D. Skroza, I. Ljubenkov, F. Burčul, and V. K. Croat, "Antioxidative/acetylcholinesterase inhibitory activity of some Asteraceae plants," Journal of Food Science and Technology, vol. 6, pp. 7-11, 2014.

[21] N. S. A. Malik and J. M. Bradford, "Recovery and stability of oleuropein and other phenolic compounds during extraction and processing of olive (Olea europaea L.) leaves," Journal of Food, Agriculture and Environment, vol. 6, no. 2, pp. 8-13, 2008.

[22] M. Ansaria, M. Kazemipourb, and S. Fatehi, "Solid-Phase Extraction and Simultaneous Determination of Tetracycline Residues in Edible Cattle Tissues Using an HPLC-FL Method," J. Iran. Chem. Soc, vol. 11, pp. 781-787, 2012.
[23] “Design- Expert- Software," 2015, http://www.statease.com.

[24] M. N. Ahmad, S. L. Liew, M. A. Yarmo, and M. Said, "Optimization of protease extraction from horse mango (Mangifera foetida Lour) kernels by a response surface methodology," Bioscience, Biotechnology and Biochemistry, vol. 76, no. 8, pp. 1438-1444, 2012.

[25] R. Wang, P. Chen, F. Jia, J. Tang, and F. Ma, "Optimization of polysaccharides from Panax japonicus C.A. Meyer by RSM and its anti-oxidant activity," International Journal of Biological Macromolecules, vol. 50, no. 2, pp. 331-336, 2012.

[26] M. B. Hossain, C. Barry-Ryan, A. B. Martin-Diana, and N. P. Brunton, "Optimisation of accelerated solvent extraction of antioxidant compounds from rosemary (Rosmarinus officinalis L.), marjoram (Origanum majorana L.) and oregano (Origanum vulgare L.) using response surface methodology," Food Chemistry, vol. 126, no. 1, pp. 339-346, 2011.

[27] C. Stévigny, L. Rolle, N. Valentini, and G. Zeppa, “Optimization of extraction of phenolic content from hazelnut shell using response surface methodology," Journal of the Science of Food and Agriculture, vol. 87, no. 15, pp. 2817-2822, 2007. 

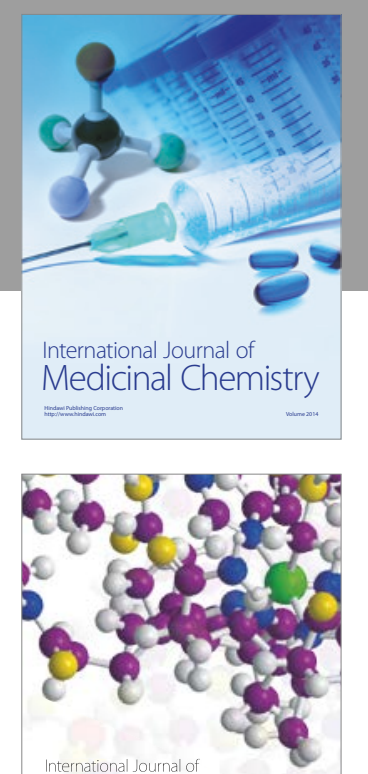

Carbohydrate Chemistry

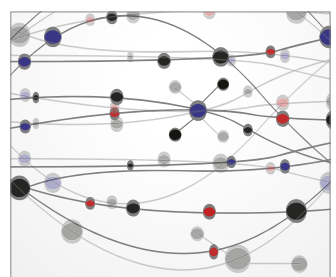

The Scientific World Journal
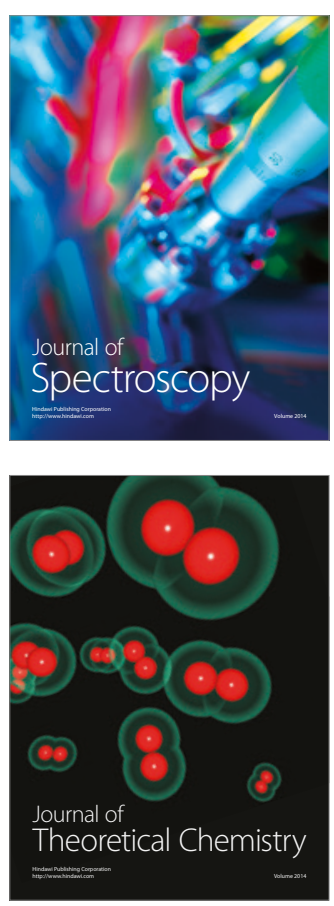
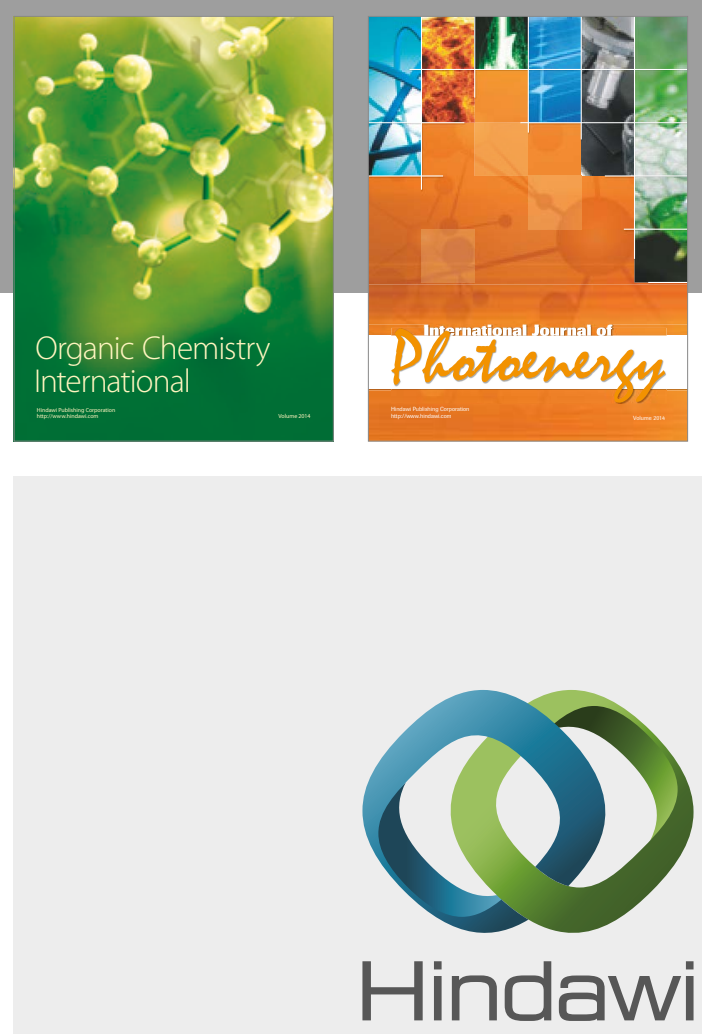

Submit your manuscripts at

https://www.hindawi.com

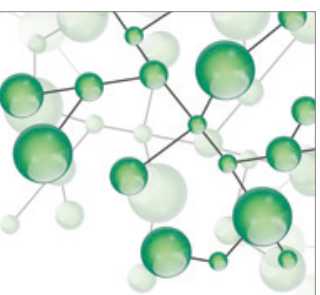

International Journal of

Inorganic Chemistry

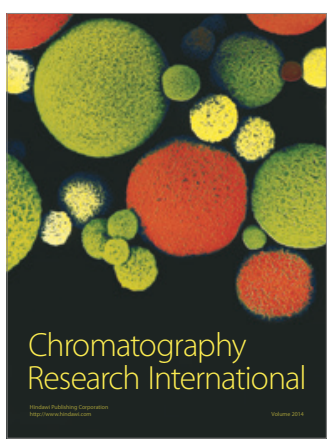

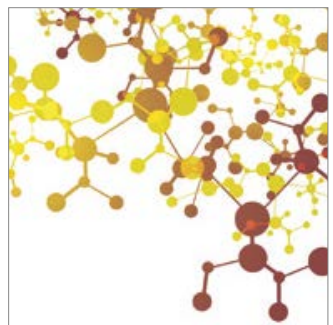

Applied Chemistry
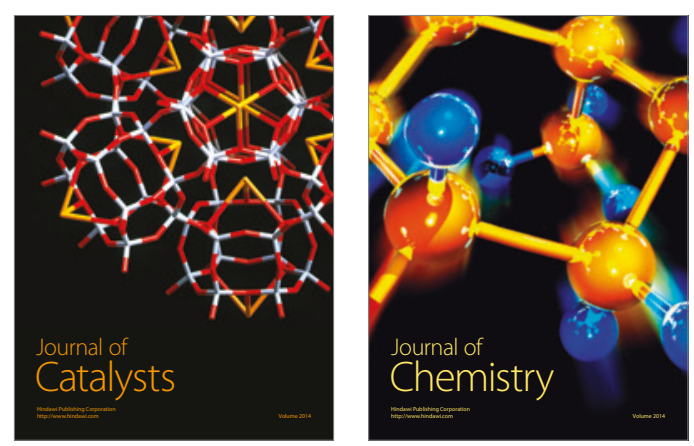
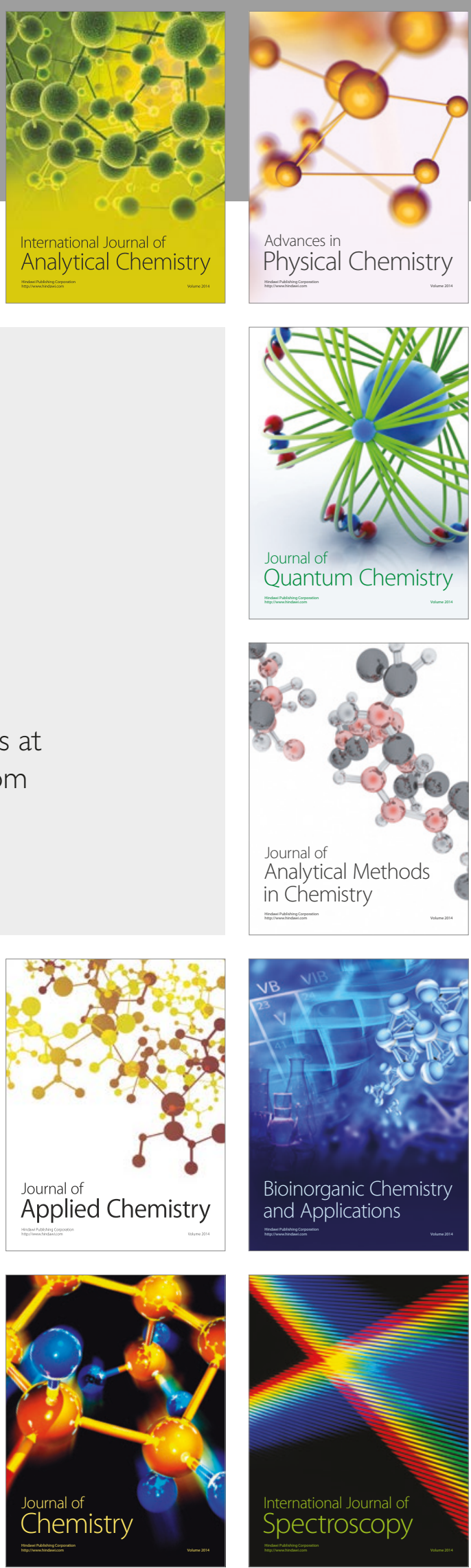